
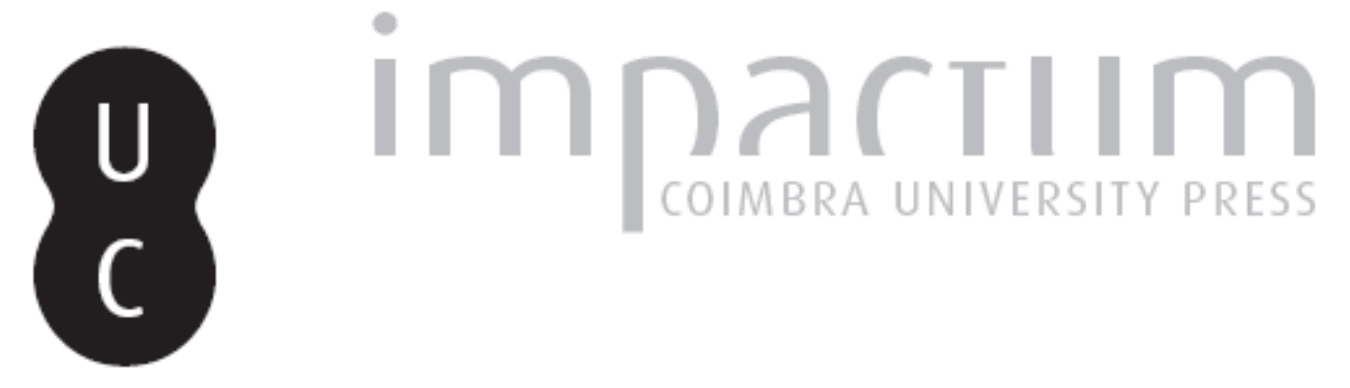

\title{
Primo approccio ad un gruppo di falere ispaniche
}

\author{
Autor(es): Baratta, Giulia
}

Publicado por: Faculdade de Letras da Universidade de Coimbra

URL persistente:

URI:http://hdl.handle.net/10316.2/37851

DOI:

DOI:http://dx.doi.org/10.14195/1647-8657_48_6

Accessed : $\quad$ 26-Apr-2023 05:47:08

A navegação consulta e descarregamento dos títulos inseridos nas Bibliotecas Digitais UC Digitalis, UC Pombalina e UC Impactum, pressupõem a aceitação plena e sem reservas dos Termos e Condições de Uso destas Bibliotecas Digitais, disponíveis em https://digitalis.uc.pt/pt-pt/termos.

Conforme exposto nos referidos Termos e Condições de Uso, o descarregamento de títulos de acesso restrito requer uma licença válida de autorização devendo o utilizador aceder ao(s) documento(s) a partir de um endereço de IP da instituição detentora da supramencionada licença.

Ao utilizador é apenas permitido o descarregamento para uso pessoal, pelo que o emprego do(s) título(s) descarregado(s) para outro fim, designadamente comercial, carece de autorização do respetivo autor ou editor da obra.

Na medida em que todas as obras da UC Digitalis se encontram protegidas pelo Código do Direito de Autor e Direitos Conexos e demais legislação aplicável, toda a cópia, parcial ou total, deste documento, nos casos em que é legalmente admitida, deverá conter ou fazer-se acompanhar por este aviso.

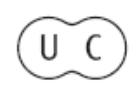


CONIMBRIGA

W.

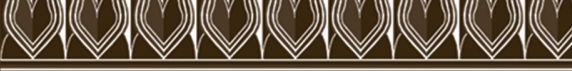

INSTITUTO DE ARQUEOLOGIA

VOLUME XLVIII • 2009

FACULDADE DE LETRAS 
Givlia BaratTa

Professore associato, Università degli Studi di Macerata

PRIMO APPROCCIO AD UN GRUPPO DI FALERE ISPANICHE

"Conimbriga" XLVIII (2009) p. 189-209

RiASSUNTO: In questo lavoro si presenta un gruppo di phalerae di cavallo di origine ispanica che costituisce il primo corpus di questa tipologia di elementi di bardatura rinvenuti nell'impero romano.

Resumo: Apresenta um conjunto de phalerae de cavalo, de origem hispânica, que constitui o primeiro corpus deste tipo de elementos de arnês achados no Império Romano. 
(Página deixada propositadamente em branco) 


\section{PRIMO APPROCCIO AD UN GRUPPO DI FALERE ISPANICHE}

Oggetto di questo breve contributo è un gruppo di falere composto, allo stato attuale, da 14 pezzi cui sino ad ora non era stato dedicato un lavoro sistematico che li comprendesse tutti ${ }^{1}$, ma che appaiono pubblicati in modo dispersivo in diverse sedi e spesso senza un approccio sufficientemente critico.

Tutti i pezzi sono realizzati in bronzo e lavorati a fusione in uno stampo con la tecnica della cera persa e presentano dimensioni piuttosto omogenee comprese tra $11 \mathrm{~cm}$ circa di altezza e $11,5 \mathrm{~cm}$ circa di larghezza².

Da un punto di vista funzionale ed estetico tutte le falere prese in esame si caratterizzano per la presenza di un elemento centrale intorno al quale si impostano quattro passanti, quasi sempre circolari. Questi sono collocati ai quattro vertici di ciascun esemplare e sono uniti tra di loro da diversi elementi decorativi che variano da pezzo a pezzo.

La parte centrale della falera può essere costituita da un elemento pieno oppure traforato. Nel primo gruppo, infatti, può trattarsi di un semplice disco leggermente convesso sulla faccia a vista della falera e concavo in quella opposta, decorato con cerchi concentrici (cat. nrr. 3, $4,10)$ oppure di un analogo elemento circolare, tendente talvolta ad una

\footnotetext{
${ }^{1}$ Per una prima raccolta incompleta di questi pezzi vedi Millán, Falera romana, pp. 197-204 e Blázquez, Historia económica, pp. 247-249, fig. 84, 1-3.

${ }^{2}$ Per diversi esemplari non sono ancora disponibili tutti i dati, in particolare le misure esatte, poiché molti musei non hanno per il momento risposto alla richiesta di informazioni sui pezzi o li ritengono irreperibili.
} 
forma più ovoidale o più simile a quella di uno scudo, interamente occupato dalla raffigurazione di un volto, maschile o femminile (cat. nrr. $5,6,8,9,11,12$ ). Tra i quattro esemplari con decorazione centrale traforata, invece, ricorre due volte il motivo del busto femminile, singolo, in un caso (cat.nr. 1), e addirittura triplice in un altro (cat. nr. 14). Gli altri due pezzi presentano, invece, il tema del cavallo (cat. nr. 2 e 7) un soggetto iconografico caratteristico del repertorio tardoantico ${ }^{3}$. In un solo caso (cat. nr. 13), infine, la decorazione centrale della falera è prettamente geometrica e risulta dall'incrocio di più elementi rettilinei che danno origine ad una serie di losanghe e triangoli.

Quattro esemplari tra quelli che presentano la decorazione centrale a disco, rispettivamente con cerchi concentrici e con la raffigurazione di volti femminili (cat. nrr. 3-6), presentano tutti la medesima tipologia di raccordo tra i passanti, costituita da un archetto con una luce a forma di serratura. Per almeno due di essi (cat. nr. 3, 4) è possibile ipotizzare la provenienza da un medesimo atelier, fermo restando che, date le estreme somiglianze formali, la realizzazione in una medesima bottega, o per lo meno in uno stesso contesto geografico e culturale, non può essere scartato, e risulta anzi fortemente probabile, anche per gli altri pezzi. Analoga considerazione va fatta per tutti gli altri esemplari per i quali, però, non si ravvisano somiglianze formali tanto evidenti da far supporre uno stesso luogo di produzione ma piuttosto una coincidenza culturale e cronologica che si evidenzia nei tratti somatici dei volti, in particolare nei grandi occhi sporgenti, nei nasi e nelle bocche oltre che nelle capigliature, nella scelta dei soggetti figurati e negli elementi decorativi punzonati in particolare sulle asole e sugli elementi strutturali e ornamentali che le uniscono.

Purtroppo i pochi dati noti circa i luoghi e i contesti di rinvenimento di queste falere non consentono di redigere una mappatura completa della loro diffusione sul territorio. Le informazioni disponibili permettono soltanto di individuare una maggiore zona di concentrazione nella parte centro-settentrionale della Penisola Iberica, corrispondente alla Tarraconense, ed una comparsa sporadica a sud in territorio betico.

\footnotetext{
${ }^{3}$ Vedi una panoramica generale in Fuentes, Bronces bajoimperiales, pp. 119-122; Aurrecoechea, Ager, Late roman iconografic representations, pp. 281-286.
} 
Le falere in questione sono verosimilmente attribuibili ad un ambiente civile più che militare, come supposto in passato $0^{4}$, legato alle grandi ville tarde, al mondo dell'allevamento dei cavalli, attività di prestigio nell'Hispania tardo antica, e alle gare circensi ${ }^{5}$. Invitano a questa ipotesi i soggetti figurati che vi compaiono, in particolare i cavalli (cat. nrr. 2 e 7), uno dei quali (cat. nr. 7) è corredato anche da una palma della vittoria e sembra inequivocabilmente associato all'ambiente delle gare circensi.

Per quanto riguarda la cronologia, questo gruppo di falere, definito da P. de Palol come "tipicas phalerae hispanorromanas", viene in genere attribuito ad età costantiniana ${ }^{6}$. Sulla base di alcuni confronti stilistici, però, è possibile proporre una datazione più tarda compresa tra la fine del IV e la prima metà del V secolo d.C. Questo inquadramento cronologico è suggerito dagli archetti con luce a forma di serratura (cfr. cat. nrr. 3-6) presenti anche su numerosi cinturoni del tipo Simancas e su avori tardi, attribuibili appunto a questo orizzonte temporale, oltre che dai tratti somatici e dalle acconciature delle figure che richiamano modelli teodosiani e preludono alle caratteristiche fisionomiche della scultura di VI-VII secolo d.C. Un ulteriore elemento a sostegno di questa datazione viene dai dati di scavo della villa di Valdanzo, da dove proviene la falera con decorazione geometrica a losanghe (cat. nr. 13), rinvenuta in un cortile attribuito alla fine del IV secolo d.C. che costituisce, dunque, un terminus post quem almeno per questo esemplare. Anche per le raffigurazioni di cavallo si possono proporre diversi confronti

\footnotetext{
${ }^{4}$ López Cuevillas, Taboada, Oppidum, p. 87; De Palol, Más bronces romanos, p. 309; Rodá, Bronces romanos, p. 83.

${ }^{5}$ Sulla diffusione e sull'importanza dei cavalli ispanici vedi Arce, Caballos, pp. 35-44; Blázquez, Aportaciones, pp. 11-46.

${ }^{6}$ De Palol Salellas, Algunas piezas de adorno de arnés, pp. 300-301. Non condivisibile è invece la successiva proposta di De Palol di attribuire il pezzo proveniente dalla provincia di Palencia (cat. nr. 11), sulla base della capigliatura della maschera, ad un arco cronologico che va da epoca flavia al primo quarto del II secolo d.C., e tantomeno la datazione della falera di San Millán (cat. nr. 5), alla fine del II-inizi del III secolo d.C., da parte di F. Lopez Cuevillas e J. Taboada sulla stessa base dell'acconciatura del mascherone che costituisce l'elemento centrale del pezzo né quella avanzata da J. Cabré Aguiló che attribuisce l'esemplare rinvenuto nella provincia di Jaén (cat. nr. 12) al I secolo d.C.
} 
iconografici e stilistici con altre rappresentazioni dell'animale presenti, ad esempio, su taluni esemplari di dischi per morsi di cavallo e su falere di diversa tipologia ${ }^{7}$, tutti databili al IV secolo d.C. anche avanzato e, comunque, non anteriori ad età costantiniana.

Per quanto concerne il loro impiego la presenza dei quattro passanti indica che vi si attaccavano altrettante corregge di pelle che si dipartivano a raggera rispetto al centro della falera come indicano le tracce di usura nell'arco di cerchio esterno dei passanti stessi, evidenti in particolare negli esemplari di Camparañon (cat. nr. 1) e di San Millán (cat. nr. 5) $)^{8}$. Per le loro dimensioni e per il peso è assai probabile che ornassero il corpo del cavallo e che fungessero da ornamento dei suoi fianchi in modo uguale o analogo a quello che si vede su di un cavallino di bronzo, elemento figurato pertinente alle sospensioni di un carro, rinvenuto a Morón de la Frontera ${ }^{9}$ (fig. 16).

\footnotetext{
${ }^{7}$ Cfr. ad esempio il pezzo di Monturque, De los Santos Gener, Falera de Monturque, pp. 30-35; gli esemplari in Blázquez, Historia económica, fig. 83-87; De Palol, Arqueologia cristiana, tav. CX; Casanovas, Rovira, Torredonjimeno, p. 50; Palol Salellas, Piezas de arnés, pp. 217-228; Aurrecoechea, Ager, Late Roman iconographic representations, pp. 281-286; Nunes Pinto, Bronzes figurativos, pp. 395-396, nr. 295, tav. 166; García y Bellido, Esculturas romanas, nr. 477, tav. 334; Salvatierra Cuenca, Arqueología visigoda, p. 50.

${ }^{8}$ Questo dato tecnico attesta inconfutabilmente che le cinghie in pelle della bardatura passavano per queste asole disposte agli angoli della falera e non negli archetti, che in taluni esemplari costitutiscono l'elemento di unione tra le asole stesse, come invece, a suo tempo, era stato supposto, vd. Lopez Cuevillas, Taboada, Oppidum, p. 87. Anche il fatto che non tutti gli esemplari presentino gli archetti ma altri elementi di congiunzione tra i passanti, che non necessariamente consentono il passaggio di eventuali corregge, dimostra ulteriormente che gli elementi funzionali di queste falere sono effettivamente le asole angolari.

${ }^{9}$ Per questo pezzo attribuito alla fine del II-inizio del III secolo d.C. vedi Blanco, Pasarriendas, pp. 99-103.
} 


\section{Catalogo}

Per la definizione della tipologia dei singoli pezzi si è tenuto conto degli elementi caratterizzanti queste falere e delle loro variabili, cui sono stati attribuiti lettere e numeri secondo le tre seguenti tabelle. La forma delle asole è indicata con una lettera maiuscola dell'alfabeto latino, il tipo di raccordo tra di esse con una cifra araba e la variante della decorazione interna con una lettera minuscola dell'alfabeto latino.

\begin{tabular}{|l|c|}
\hline \multicolumn{2}{|c|}{ asole } \\
\hline tonde & $\mathrm{A}$ \\
\hline triangolari & $\mathrm{B}$ \\
\hline
\end{tabular}

\begin{tabular}{|l|l|}
\hline \multicolumn{2}{|c|}{ raccordi tra le asole } \\
\hline tratto verticale & 1 \\
\hline archi & 2 \\
\hline cerchi & 3 \\
\hline pelte & 4 \\
\hline esse & 5 \\
\hline trangoli & 6 \\
\hline quadrati & 7 \\
\hline volti & 8 \\
\hline
\end{tabular}

\begin{tabular}{|l|l|}
\hline \multicolumn{2}{|c|}{ elemento interno } \\
\hline pieno con decorazione geometrica & $\mathrm{a}$ \\
\hline pieno con decorazione figurata & $\mathrm{b}$ \\
\hline traforato con decorazione geometrica & $\mathrm{c}$ \\
\hline traforato con busto & $\mathrm{d}$ \\
\hline traforato con cavallo & $\mathrm{e}$ \\
\hline
\end{tabular}


Nr. 1, Fig. 1a-b

Luogo di rinv. Camparañon (Soria)

Luogo di cons. Museo Numantino

Misure

h. $10 \mathrm{~cm}$; larg. $9 \mathrm{~cm}$

La falera è realizzata con la tecnica della cera persa. La parte centrale è concava sul rovescio e convessa sulla faccia a vista. I quattro passanti circolari, posizionati agli angoli della falera, presentano una decorazione punzonata con cerchietti e piccolo punto centrale, tipica della toreutica tardoantica ${ }^{10}$. I passanti sono raccordati a destra e sinistra da due bastoncini con andamento verticale sui quali si ripete la stessa decorazione. L'elemento centrale è costituito da un busto femminile adornato da un pendente al collo e con veste panneggiata. I suoi tratti denotano un'evidente perdita di naturalismo ed una certa ieraticità. L'ampia capigliatura, coronata da una treccia, caratterizza i ritratti femminili sin da epoca costantiniana per essere poi ripresa in epoca teodosiana e continuare senza troppe variazioni sino alla fine del $\mathrm{V}$ secolo d.C. ${ }^{11}$. L'acconciatura funge da raccordo superiore tra i passanti mentre la parte inferiore del busto serve da congiunzione tra le asole nella parte inferiore.

Tipo A-1-d

Dat. Fine IV secolo - prima metà del V secolo d.C. Secondo Millán il pezzo si daterebbe al III secolo d.C. per i caratteri stilistici che riprenderebbero modelli celtici ancora vivi nelle zone non troppo romanizzate della Penisla Iberica mentre Pascual Díez la attribuisce al IV secolo d.C.

\footnotetext{
${ }^{10}$ Vedi alcuni esempi in Blázquez, Historia económica, tav. 83, nrr. 1, 2, 4; tav. 84, nr. 4, tav. 87, nr. 1; Salvatierra Cuenca, Arqueología visigoda, p. 50

${ }^{11}$ Cfr. Delbrück, Spätantike Kaiserporträts, pp. 46-52 in part. due ritratti privati della metà del IV secolo d.C. ove la treccia assume proporzioni notevoli (fig. 19 e 20) e i più tardi ritratti del tipo Flacilla (tavv. 99-101) e p. 52 ove l'autore scrive "Die Frisur hat von jetzt ab eine Normalform, fast ohne Variationen, und bleibt in beiden Reichshälften bis gegen das Ende des 5. Jh.s. gleich". Vedi anche Calza, Iconografia romana imperiale, pp. 264-270, ritratti di Costanza I (314-324) nrr. 178-182 e Marcos, Política dinástica, pp. 155-164, part. fig. 4 .
} 
Bibl. Taracena Aguirre, Carta arquéologica España, p. 48; Millán, Falera romana, pp. 197-198, tav. XXI, 1; De Palol, Más bronces romanos, p. 309; Pascual Díez, Carta Arqueológica Soria, pp. 70-71, fig. 35 .

\section{Nr. 2, Fig. 2a-b}

Luogo di rinv. Palencia, Plazuela de San Lázaro

Luogo di cons. Ex collezione privata Fontaneda, Castillo de Ampudia, Palencia
Misure
h. $7,8 \mathrm{~cm}$; larg. $8 \mathrm{~cm}$

La falera è realizzata a stampo con la tecnica della cera persa. I quattro passanti circolari sono untiti tra di loro da elementi rettilinei con una decorazione incisa a spina di pesce. Al centro della falera è raffigurato di profilo un cavallo con le quattro zampe idealmente poggiate sul terreno reso con un elemento verticale che a sua volta collega i passanti inferiori. Il cavallo costituisce un tema iconografico estremamente frequente sia sugli elementi di bardatura dei cavalli stessi che sulle parti metalliche dei carri e sui cinturoni di epoca tardo-antica particolarmente in Hispania $^{12}$.

Tipo A-1-e

Dat. Seconda metà del IV - prima metà del V secolo d.C.

Bibl. De Palol, Más bronces romanos, p. 309 e 311, tav. III, 1; Blázquez, La caballeria, p. 65, fig. 5; Blázquez, Historia económica, fig. 84,1

Nr. 3, Fig. 3

Luogo di rinv. provenienza non precisata

Luogo di cons. MuseoValencia de Don Juan

Misure non disponibili

La falera è realizzata a fusione mediante la tecnica della cera persa. La parte posteriore, in particolare il disco centrale, risultano concavi mentre la faccia a vista è convessa. Le quattro asole circolari sono unite

\footnotetext{
${ }^{12}$ Vedi supra nota nr. 3. Per alcuni confronti senza pretesa di completezza vedi ad esempio Aurrecoechea Fernández, Cinturones romanos, p. 73, nr. 7, p. 115, figg. 1, 3, 6 che data questi pezzi alla fine del IV secolo d.C.
} 
tra di loro rispettivamente da due elementi ad arco con una luce a forma di serratura per la presenza di una sporgenza che chiude la parte sommitale arcuata quasi fosse la semplificazione di un porticato con colonne coronate da capitelli ed unite da archi. Questi archetti di raccordo trovano uno stringente confronto con alcuni cinturoni del tipo Simancas rinvenuti in contesti attribuibili alla fine del IV-inizi del V secolo d.C. ${ }^{13}$, in un'area geografica che coincide con quella di diffusione di alcune delle nostre falere e su avori tardi di V secolo ${ }^{14}$. L'elemento centrale della falera è costituito da un disco decorato da linee incise concentriche.

Tipo A-2-a

Dat. Fine IV - prima metà del V secolo d.C.

Bibl. Millán, Falera romana, p. 203, tav. XXII, 2; De Palol, Más bronces romanos, p. 310 .

Nr. 4

Luogo di rinv. non meglio precisata provenienze ispanica

Luogo di cons. Madrid, Museo Arqueológico Nacional

Misure non disponibili

La falera è sostanzialmente uguale alla precedente tanto da non potersi escludere che siano state realizzate entrambe nel medesimo atelier.

Tipo A-2-a

Dat. Fine IV - V secolo d.C. Secondo Millán il pezzo sarebbe visigota.

Bibl. Millán, Falera romana, pp. 203-204, tav. XXII, 3; De Palol Salellas, Algunas piezas de adorno de arnés, fig. 1, A; De Palol, Bronces romanos de Palencia, p. 237; De Palol, Más bronces romanos, p. 310.

\footnotetext{
${ }^{13}$ Cfr. Aurrecoechea Fernández, Cinturones romanos, p. 37, fig. 12, e p. 94, nr. 98, e p. 90, nr. 80, p. 94, nr. 100 e pp. 155-163; Aurrecoechea, Roman Military equipment 7, 1996, fig. 21

${ }^{14}$ Bettini, Scultura bizantina, p. 44.
} 


\section{Nr. 5, Fig. 5}

Luogo di rinv. Cidá do Castro, San Millán (Orense), tra pietrame di spoglio

Luogo di cons. Museo Arqueológico Provincial de Orense, cat. nr. 3487
Misure
h. $9,5 \mathrm{~cm}$; larg. $8,6 \mathrm{~cm}$

La falera è realizzata a stampo con la tecnica della cera persa e presenta la parte posteriore leggermente concava e quella a vista convessa. Come già nei due pezzi precedenti le asole circolari sono unite tra di loro rispettivamente da due elementi ad arco con una luce a forma di serratura ${ }^{15}$ che, per i confronti esistenti, permettono un inquadramento cronologico tra le fine del IV secolo d.C. e l'inizio di quello seguente. L'elemento centrale è composto da un disco la cui superficie è interamente occupata dalla raffigurazione di un volto femminile dai tratti somatici piuttosto grossolani ${ }^{16}$. L'ampia capigliatura, resa con varie file di tratti a spina di pesce per rendere l'ondulazione dei capelli nella parte anteriore, e coronata da una treccia, caratterizza i ritratti femminili sin da epoca costantiniana per essere poi ripresa in epoca teodosiana e continuare, senza troppe variazioni, sino alla fine del V secolo d.C. ${ }^{17}$.

Tipo A-2-b

Dat. Fine IV - V secolo d.C. López Cuevillas e Taboada, invece, sulla base della capigliatura, sostengono un inquadramento cronologico tra la fine del II- e gli inizi III del secolo d.C., mentre gli editori del catalogo "Bronces romanos" propongono una datazione al III secolo o più tarda.

Bibl. López Cuevillas, Taboada, Oppidum, pp. 86-88, fig. 14; De Palol, Bronces romanos de Palencia, pp. 236-237; De Palol, Más bronces romanos, p. 310; Rodríguez Colmenero, Galicia Meridional, p. 323, tav. III, 5; AA.VV., Bronces romanos, p. 202, nr. 73.

\footnotetext{
${ }^{15}$ Per questi elementi ad arco vedi supra note 13 e 14.

${ }^{16}$ Il volto umano costituisce un soggetto decorativo frequente nel campo della metallurgia tardo antica, cfr. Aurrecoechea Fernández, Cinturones romanos, p. 113.

${ }^{17}$ Vedi supra nota 11.
} 


\section{Nr. 6, Fig. 6}

Luogo di rinv. Sconosciuto

Luogo di cons. Museo di Santarém. Probabilmente perduto

Misure non disponibili

La falera è mancante di quasi tutta la parte superiore e di quella inferiore dove, secondo quanto è possibile ricostruire, le asole erano unite da un solo elemento ad arco con luce a forma di serratura del tutto analogo a quello delle tre falere precedentemente descritte, mentre lungo i lati gli archetti dovevano essere tre. Il campo centrale quadrangolare, concavo nella parte posteriore e convesso in quella a vista, è quasi interamente occupato dalla raffigurazione di un volto femminile con ampia capigliatura apparentemente con scriminatura centrale.

Tipo A-2-b

Dat. Fine IV - V secolo d.C. Palol, sulla base della capigliatura, propone una datazione ad epoca severiana.

Bibl. De Palol, Bronces romanos de Palencia, p. 237, fig. 1, 5; Pinto, Bronzes figurativos, pp. 393-394, nr. 293, tav. 165.

\section{Nr. 7, Fig. 7a-b}

Luogo di rinv. Sconosciuto

Luogo di cons. Collezione privata

Misure h. 10,8 cm; larg. 9,9 cm

La falera è realizzata a stampo con la tecnica della cera persa. La parte posteriore è concava e quella a vista è convessa. I passanti circolari sono congiunti tra di loro, nella parte superiore ed inferiore, da un elemento a $\mathrm{T}$ che da origine a due luci di forma quadrilatera irregolare, mentre a destra e a sinistra sono uniti da due elementi ad S sovrapposti. Nel campo centrale della falera, chiuso da una cornice circolare decorata con tralci di vite ed elementi a spina di pesce incisi, che forse richiamano il tema della palma della vittoria, è raffigurato, di profilo, un cavallo con la zampa anteriore sinistra alzata, secondo un motivo iconografico estremamente frequente in epoca tardo antica sugli elementi di bardatura dei cavalli, su quelli decorativi dei carri e sui cinturoni ${ }^{18}$. Sulla sua coscia

\footnotetext{
${ }^{18}$ Per alcuni confronti vedi ad esempio il pezzo di Monturque, De los Santos Gener, $L a$ falera de Monturque, pp. 30-35; gli esemplari in Blázquez, Historia económica, fig.
} 
posteriore destra è presente un cristogramma racchiuso in un cerchio, mentre sulla corrispondente zampa anteriore è incisa una palma della vittoria. Tra questi, lungo tutto il fianco dell'animale, corre l'iscrizione $M / A V E$ riferibile al nome del cavallo o a quello del suo proprietario.

Tipo A-3-7-e

Dat. Seconda metà del IV secolo d.C.-inizio del V secolo d.C.

Bibl. Baratta, Ornamento iscritto.

Nr. 8 , Fig. 8

Luogo di rinv. nel territorio di Segovia

Luogo di cons. Museo Valencia de Don Juan

Misure non disponibili

In questa falera i quattro passanti circolari sono uniti tra di loro rispettivamente da un elemento a forma di pelta. Il campo centrale a forma di scudo è interamente occupato dalla raffigurazione di un volto apparentemente maschile e con i tratti somatici piuttosto grossolani.

Tipo A-4-b

Dat. seconda metà del IV secolo d.C.-inizio V secolo d.C.

Bibl. Millán, Falera romana, pp. 197, tav. XXI, fig. 2; De Palol, Más bronces romanos, p. 310; Blázquez, Historia económica, fig. 84, 2

\section{Nr. 9, Fig. 9a-b}

Luogo di rinv. Sconosciuto

Luogo di cons. Collezione Vigueira a Villagarcía de Arosa Misure non disponibili

La falera è realizzata a stampo con la tecnica della cera persa. I quattro passanti sono uniti nella parte inferiore e lungo i lati della falera rispettivamente da un elemento a forma di pelta. Nella parte superiore, invece, la congiunzione è assicurata da un elemento triangolare, fiancheggiato da due piccoli dischi, che nasce direttamente dalla cornice

83-87; De Palol, Arqueología cristiana, tav. CX; Casanovas, Rovira, Torredonjimeno, p. 50; Palol Salellas, Piezas de arnés, pp. 217-228; Aurrecoechea, Ager, Late roman iconografic representations, pp. 281-286; Nunes Pinto, Bronzes figurativos, pp. 395396, nr. 295, tav. 166; García y Bellido, Esculturas romanas, nr. 477, tav. 334. 
che abbraccia il campo centrale di forma ovoidale. Questo è interamente occupato dalla raffigurazione di un volto apparentemente maschile dai tratti somatici molto grossolani e con grandi occhi piangenti a forma di mandorla.

Tipo A-4-b

Dat. Fine IV-inizio del V secolo d.C.

Bibl. Millán, Falera romana, p. 197, fig. 1; De Palol, Más bronces romanos p. 310, tav. III, 2; Blázquez, Historia económica, fig. 84,2

Nr. 10, Fig. 10

Luogo di rinv. Julióbriga

Luogo di cons. Già Museo Cantábrico di Comillas

Misure non disponibili

I quattro passanti lungo due lati contrapposti sono uniti tra di loro rispettivamente da due elementi a forma triangolare, mentre i restanti due lati non presentano alcuna congiunzione diretta tra le asole. Il campo centrale circolare è decorato da una serie di linee concentriche incise.

Tipo A-6-a

Dat. Fine IV - V secolo d.C.

Bibl. Millán, Falera romana, p. 200, fig. 2

Nr. 11, Fig. 11

Luogo di rinv. Provincia di Palencia, non lontano dalla zona di Aguilar-Herrera de Pisuerga

Luogo di cons. Collezione privata Fontaneda, ora Castillo de Ampudia, Palencia?

Misure non disponibili

La falera è realizzata a stampo con la tecnica della cera persa. È mancante della metà inferiore del bordo sinistro e della porzione sinistra della parte inferiore. Si caratterizza per avere i quattro passanti uniti lungo ciascuno dei lati rispettivamente da un elemento di forma triangolare. Lungo il bordo esterno di ciascuno dei passanti è presente una piccola sporgenza di carattere decorativo. Sia le asole che i triangoli sono decorati con piccoli tratti semicircolari incisi secondo una moda 
diffusa nella toreautica tardoantica ${ }^{19}$. Il campo centrale, di forma ovoidale, è interamente occupato dalla raffigurazione di un volto con un'alta capigliatura che scende oltre le orecchie ed è resa con una serie di tratti paralleli ad indicare le ciocche dei capelli. La sua conformazione, probabilmente esasperata per adattarsi alla forma dell'elemento mediano della falera, ricorda acconciature diffuse a partire da Valentiniano I, in epoca teodosiana e anche successivamente, pur con variazioni per quanto riguarda le ciocche lateriali e l'altezza dei capelli della parte frontale, almeno sino a Marciano ${ }^{20}$. Capigliature analoghe sono attestate anche sui dittici di Areobindo, Clementino, Anastasio e su quello ancora più tardo di Basilio ${ }^{21}$.

\section{Tipo A-6-b}

Dat. Fine IV - V secolo d.C anche avanzato. Secondo Palol, invece, il pezzo andrebbe attribuito ad un arco di tempo compreso tra l'epoca flavia e il primo quarto II secolo d.C.

Bibl. De Palol, Bronces romanos de Palencia, p. 236, tav. IV, 1; De Palol, Más bronces romanos, p. 310; Blázquez, Historia económica, p. 84, nr. 3 .

Nr. 12, Fig. 12a-b

Luogo di rinv. Provincia di Jaén

Luogo di cons. Museo Arqueológico Nacional, Madrid

Misure non disponibili

In questo esemplare l'elemento di unione tra i quattro passanti è costituito da altrettanti volti disposti rispettivamente uno al centro di ciascun lato. Il campo centrale di forma irregolare è quasi interamente occupato dalla raffigurazione di un viso fortemente aggettante che ne risparmia soltanto il perimetro. Tutti i volti della falera presentano una capigliatura corta con ciocche di capelli rese con tratti incisi.

\footnotetext{
${ }^{19}$ Vedi supra nota nr. 10.

${ }^{20}$ Delbrück, Spätantike Kaiserporträts, pp. 43-46. Vedi anche Kollwitz, Oströmische Plastik, pp. 114-131, Stichel, Römische Kaiserstatue e L'Orange, Spätantikes Porträt, p. 149, nr. 123, fig. 229 in particolare oltre che per la capigliatura anche per la forma estremamente allungata della calotta cranica.

${ }^{21}$ Ravegnani, Consoli e dittici consolari, pp. 134-142 e pp. 157-158.
} 


\section{Tipo A-8-b}

Dat. Fine IV - V secolo d.C. Secondo J. Cabré Aguiló il pezzo si daterebbe al I secolo d.C. e sarebbe un oggetto d'importazione giunto via mare.

Bibl. Cabré Aguiló, Tesoro, p. 289, fig. 9; Millán, Falera romana, p. 200, fig. 1; De Palol, Más bronces romanos, p. 310.

\section{Nr. 13, Fig. 13a-b}

Luogo di rinv. Da una villa a Valdanzo, Soria (contesto archeologico IV-V secolo d.C.)

Luogo di cons. Non indicato

Misure h. $9 \mathrm{~cm} ;$ larg. 7,5

La falera è realizzata con la tecnica della cera persa. La parte posteriore risulta leggermente concava rispetto alla faccia a vista che è piana. La falera si caratterizza per la presenza su due lati contrapposti rispettivamente di una fila di tre passanti ciascuna. La parte centrale è invece decorata da un motivo geometrico ottenuto dall'intreccio di elementi rettilinei con andamento obliquo che danno origine a luci di forma triangolare e romboidale. Su tutta la superficie sono punzonati elementi decorativi circolari, tipici della toreutica tardo antica ${ }^{22}$, che risultano mancanti sulla metà esterna delle asole d'angolo e su una porzione, sempre esterna, di quelle centrali. Come nel caso della falera di Camparañon (cat. nr. 1) l'assenza della decorazione sembra dovuta all'usura del metallo e costituisce, dunque, un utile indizio rigurado al posizionamento della falera sul corpo del cavallo e all'andamento delle corregge di pelle che passavano nelle asole.

Tipo A-1-3-c

Dat. Seconda metà IV - V secolo d.C. Secondo A. Jimeno Martínez, J.L. Argente Oliver, J. Gómez Santa Cruz il pezzo sarebbe pertinente alla seconda metà del IV secolo d.C., datazione con cui concorda anche A.C. Pascual Díez

Bibl. Jimeno Martínez, Argente Oliver, Gómez Santa Cruz, Villa de San Pedro, p. 441, fig. 18, 241, tav. VI, 2; Pascual Díez, Carta Arqueológica Soria, p. 70, nota 77.

\footnotetext{
${ }^{22}$ Per alcuni confronti vedi supra nota nr. 10.
} 
Nr. 14, Fig. 14

Luogo di rinv. Largo Ferreira Caetano, Chaves $560^{23}$

Luogo di cons. Chaves, Museu da Região Flaviense, inv. nr.

Misure

H. $10 \mathrm{~cm}$; larg. $11,4 \mathrm{~cm}$, peso $346 \mathrm{gr}$.

La falera realizzata a stampo con la tecnica della cera persa è concava nella parte posteriore e convessa in quella a vista. Lungo i lati un elemento verticale con decorazione esterna dentellata e rispettivamente un grande volto femminile a sinistra ed uno maschile a destra funge da unione tra i passanti. Entrambi i volti presentano tratti somatici piuttosto grossolani con occhi fortemente sporgenti. Le capigliature, le sopracciglia e la barba sono rese con profondi solchi incisi. La parte centrale della falera presenta tre figure femminili prive di abbigliamento disposte a piramide. Quella superiore, visibile sino all'inguine, reca nella sua mano destra un oggetto attualmente di difficle lettura, forse un marsupium. Le altre due sono raffigurate a mezzo busto. Tutte sono caraterizzate da tratti somatici molto grossolani e da capigliature con scriminatura centrale rese con profondi tratti incisi.

Tra i passanti della falera, nella parte superiore, si trova un elemento ad arco all'interno del quale si inserisce la testa della figura femminile superiore, mentre il limite inferiore si chiude con un elemento orizzontale decorato con tratti obliqui profondamente incisi.

Tipo A-1-2-8-d

Dat. fine IV secolo - prima metà del V secolo d.C.

Bibl. Rodríguez Colmenero, Galicia Meridional, p. 223, tav. VI, 1; Pinto, Bronzes figurativos, p. 393, nr. 292, tav. 165.

\section{Falere simili}

Nr. 15, Fig. 15

Luogo di rinv. Non precisato

Luogo di cons. Collezione Lázaro Galdiano

Misure non disponibili

\footnotetext{
${ }^{23}$ Ringrazio il Dr. António Ramos e il Departamento Sócio Cultural della Câmara Municipal di Chaves per avermi gentilmente fornito informazioni sulla falera e diverses fotografie del pezzo.
} 
La falera è realizzata a stampo con la tecnica della cera persa. Al centro della parte superiore ed inferiore e di quelle laterali sono collocati degli occhielli di forma rettangolare allungata uniti tra di loro dai corpi di altrettanti leoni raffigurati di profilo. Questi generano ai quattro angoli esterni della falera quattro asole di forma triangolare con gli spigoli arrotondati. La parte centrale, circolare, è quasi interamente occupata dalla testa di una medusa in forte rilievo, un soggetto con valore apotropaico frequente nel repertorio iconografico di epoca romana, che ne risparmia solo il contorno destinato a fungere da cornice.

Il pezzo per la sua conformazione è apparentemente simile a quelli caratterizzati dai passanti circolari in posizione angolare, tanto che Millán, nel primo repertorio dedicato a questa tipologia di falere, vi aveva incluso anche questo esemplare. In questo caso però la presenza di elementi decorativi esterni applicati alle asole angolari fa sospettare che non fossero destinate al passaggio delle corregge di cuoio della bardatura del cavallo, funzione per la quale si prestano molto meglio le quattro luci rettangolari che si trovano lungo i lati e nella parte superiore ed inferiore della falera stessa. Se così fosse, come appare abbastanza probabile, questo esemplare avrebbe solo una somiglianza formale con quelli caratterizzati dai passanti angolari mentre ne differirebbe per le modalità di collocazione nell' ambito della bardatura del cavallo. Infatti, in questo caso, i passanti non sarebbero disposti ad $\mathrm{X}$ rispetto al centro della falera bensì a croce. Il pezzo in questione, pertanto, troverebbe un confronto diretto, non solo stilistico ed iconografico ma anche funzionale, in una falera conservata al Museo Nacional de Arqueología di Tarragona (inv. nr. 4743) 24 $^{24}$ datata al II-III secolo d.C.

Dat. III-IV secolo d.C.

Bibl. Millán, Falera romana, pp. 198-199, tav. XXI, 3

\footnotetext{
${ }^{24}$ Mayer, Roma a Catalunya, pp. 28-29. Vedi anche un pezzo funzionalmente analogo conservato al Museo di Rabat, Boube-Piccot, Bronzes antiques du Maroc III, p. 237, nr. 382 , tav. 83 .
} 


\section{ABBREVIAZIONI BIBLIOGRAFICHE}

AA.VV., Bronces romanos = AA.VV., Los Bronces romanos en España. MayoJulio 1990, Palacio de Velazquez Parque del Retiro, Madrid 1990.

Arce, Caballos = J. Arce, Los caballos de Símmaco, in Faventia 4, 1, 1982, pp. 35-44.

Aurrecoechea Fernández, Cinturones romanos = J. Aurrecoechea Fernández, Los cinturones romanos en la Hispania del Bajo Imperio (Monographies Instrumentum 19), Montagnac 2001.

Aurrecoechea, Ager, Late roman iconografic representations $=\mathrm{J}$. AurrecoecheA, B. Ager, Late roman iconografic representations on Hispano-Roman bridle cheek-pieces, in BJb 200, 2000, pp. 281-286.

BARATTA, Ornamento iscritto $=\mathrm{G}$. BARATTA, Un ornamento iscritto pertinente a una bardatura di cavallo, in M. L. CALDELli, G. L. GREgori, S. OrLANDI (curr.), Epigrafia 2006. Atti della XVIe rencontre sur l'épigraphie in onore di Silvio Panciera con altri contributi di colleghi, allievi e collaboratori, III (Tituli 9), Roma 2008, pp. 13.

BetTini, Scultura bizantina $=$ S. BetTINI, La scultura bizantina, vol. I, Firenze 1944.

Blanco, Pasarriendas = A. Blanco, El pasarriendas romano de Morón, in AEspA 40, 1967, pp. 99-103.

BlÁzQuez, Aportaciones = J.M. Blázquez, Aportaciones al estudio de la España romana en el Bajo Impero (Colección Fundamentos 105), Madrid 1990.

BlÁzQuez, Caballería = J.M. BlÁzQuEz, La caballería en Hispania durante el Bajo Imperio, in Hestiasis. Studi di tarda antichità offerti a Salvatore Calderone (Studi Tardoantichi 2), Messina 1986, pp. 45-76.

BlÁzQuez, Historia económica = J.M. BlázQuez, Historia económica de la Hispania Romana, Madrid 1978.

Boube-Piccot, Bronzes antiques du Maroc $I I I=$ Chr. Boube-Piccot, Les bronzes antiques du Maroc III. Les chars et l'attelage (Études et travaux d'archéologie marocaine 8), Rabat 1980.

CABré Aguiló, Tesoro = J. CABré Aguiló, El tesoro de Chão de Lamas Miranda do Corvo (Portugal), in Actas y Memorias de la Sociedad Española de Antropologia, Etnografia y Prehistoria 6, 1927, pp. 271-289.

CALzA, Iconografia romana imperiale $=\mathrm{R}$. CALZA, Iconografia romana imperiale da Carausio a Giuliano (287-363 d.C.) (Quaderni e guide di archeologia III), Roma 1972.

Casanovas, Rovira, Torredonjimeno = A. Casanovas, J. Rovira (edd.), Torredonjimeno. Tresor, Monarquia i litúrgia. Exposició Novembre 2003Febrer 2005, Barcelona 2003. 
De los Santos Gener, La falera de Monturque = S. De los Santos Gener, La falera de Monturque, in Memorias de los Museos Arqueológicos Provinciales 11-12, 1950-51, pp. 30-35.

De Palol Salellas, Algunas piezas de adorno de arnes = P. De Palol Salellas, Algunas piezas de adorno de arnés de época tardorromana e hispanovisigoda, in AEspA 25, 1952, pp. 297-319.

De Palol, Bronces romanos de Palencia $=$ P. DE PAlol, Bronces romanos de la provincia de Palencia, in BSAA 33, 1967, pp. 236-240.

De Palol, Arqueología cristiana = P. De Palol, Arqueología cristiana de la España Romana (Monumentos I), Madrid, Valladolid 1967.

De Palol, Más bronces romanos $=\mathrm{P}$. De Palol, Más bronces romanos de la provincia de Palencia, in BSAA 34-35, 1969, pp. 308-312.

DelBRÜCK, Spätantike Kaiserporträts = R. DelBRÜCK, Spätantike Kaiserporträts von Constantinus Magnus bis zum Ende des Westreiches (Studien zur spätantiken Kunstgeschichte 8), Berlin, Leipzig 1933.

FuENTES, Bronces bajoimperiales = A. FuENTES, Los bronces bajoimperiales en Hispania, in AA.VV., Los bronces romanos en España, Madrid 1990, p. 119-122.

García y Bellido, Esculturas romanas $=$ A. García y Bellido, Esculturas romanas de España y Portugal, Madrid 1949.

Jimeno Martínez, Argente Oliver, Gómez Santa Cruz, Villa de San Pedro= A. Jimeno Martínez, J.L. Argente Oliver, J. Gómez Santa Cruz, La "villa" de San Pedro de Valdanzo, in Zephyrus 41-42, 1988-89, pp. 419-454.

Kollwitz, Oströmische Plastik = J. Kollwitz, Oströmische Plastik der theodosianischen Zeit (Studien zur spätantiken Kunstgeschichte 12), Berlin 1941.

L'Orange, Spätantikes Porträt = H.P. L'Orange, Studien zur Geschichte des spätantiken Porträts (Instituttet for sammenlignende Kulturforskning serie B, skrifter 23), Oslo, Leipzig, Paris, London, Cambridge Mass. 1933.

López Cuevillas, Taboada, Oppidum = F. López Cuevillas, J. Taboada, Un oppidum de la tribu de los Bibalos, in AEspA 28, 1955, pp. 69-89.

Marcos, Política dinástica $=$ M. Marcos, Política dinástica en la corte de Teodosio I: Las imágenes de Aelia Flavia Flacilla, in R. TejA, C. PÉrez (edd.), Actas congreso internacional "La Hispania de Teodosio”, Salamanca 1997.

Mayer, Roma a Catalunya = M. MAYer (ed.), Roma a Catalunya, Barcelona 1992.

Millán, Falera romana = C. Millán, Falera romana de Camparañón, in Actas y Memorias de la Sociedad Española de Antropología, Etnografía y Prehistoria 22, 1947, pp. 797-204. 
Palol Salellas, Piezas de arnés $=$ P. Palol Salellas, Dos piezas de arnés con representaciones de caballos (Museo de Linares e Instituto de Estudios Giennenses), in Oretania 2, 5, 1960, pp. 217-228.

Pascual Díez, Carta Arqueológica Soria = A.C. Pascual Díez, Carta Arqueológica Soria. Zona Centro, Soria 1991.

PInTo, Bronzes figurativos = A. J. NunEs PINTO, Bronzes figurativos romanos de Portugal, Coimbra 2002.

Ravegnani, Consoli e dittici consolari = E. Ravegnani, Consoli e dittici consolari nella tarda antichità, Roma 2006.

RodÁ, Bronces romanos = I. RodÁ, Bronces romanos de la Hispania citerior, in AA.VV., Los bronces romanos en Hispania, Madrid 1990, p. 83.

Rodríguez Colmenero, Galicia Meridional = A. Rodríguez Colmenero, Galicia Meridional Romana, Universidad de Deusto, 1977.

Salvatierra Cuenca, Arqueología visigoda = V. Salvatierra Cuenca, L'arqueología visigoda a Jaén, in CASAnovas, Rovira, Torredonjimeno, pp. 45-52.

STICHEL, Römische Kaiserstatue = R. H. W. STICHEL, Die römische Kaiserstatue am Ausgang der Antike. Untersuchungen zum plastischen Kaiserporträt seit Valentinian I. (364-375 v. Chr.) (Studia Archaeologica 24), Roma 1982

Taracena Aguirre, Carta arqueológica España= B. Taracena Aguirre, Carta arqueológica de España, Madrid 1941. 
Est. I

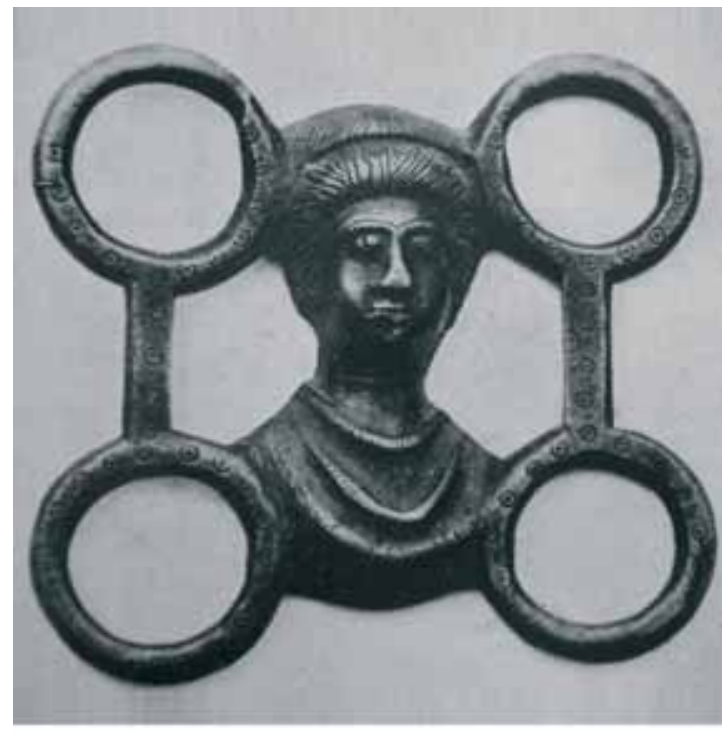

FIG. 1a-Nr. 1 da Millán, Falera romana, tav. XXI, 1

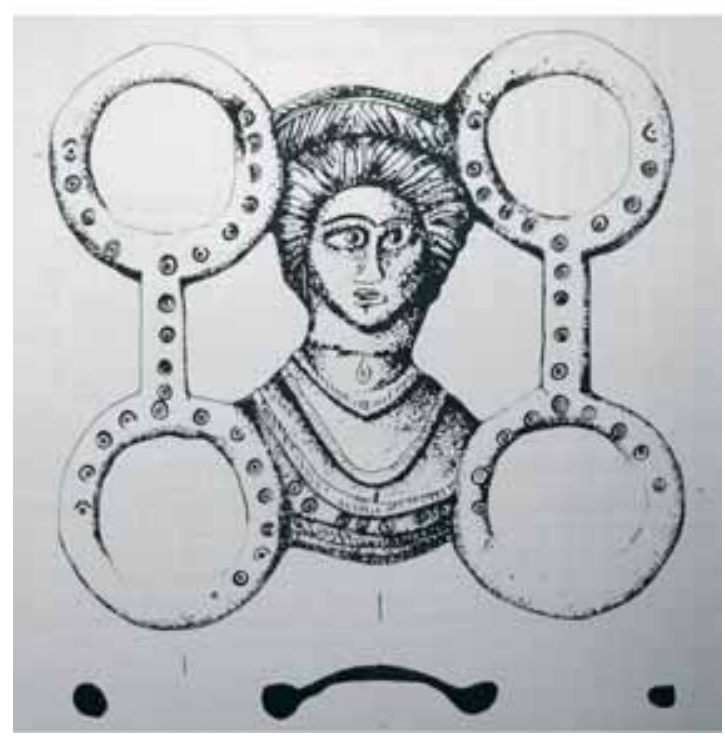

Fig. 1b - Nr. 1 da Pascual Díez, Carta Arqueológica Soria, fig. 35 
Est. II

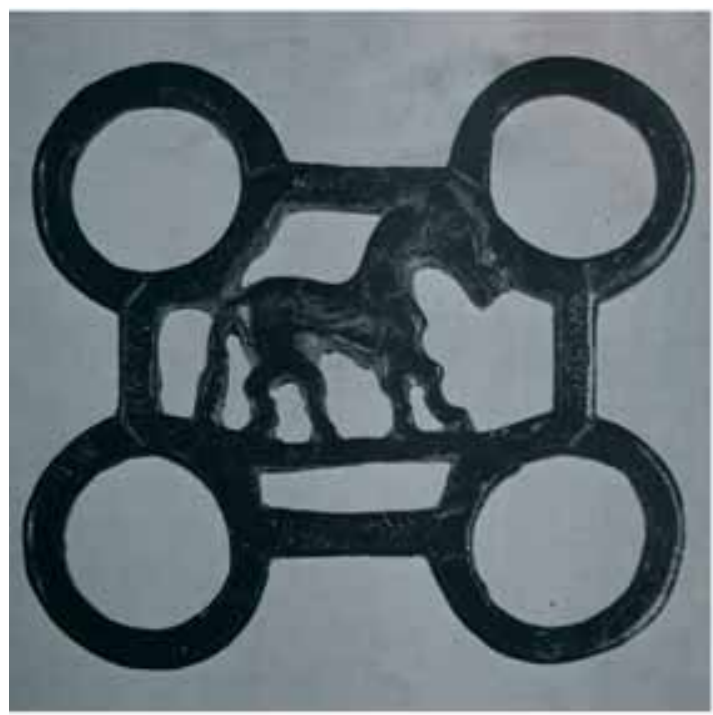

Fig. 2a - Nr. 2 da Palol, Más bronces romanos, tav. III, 1

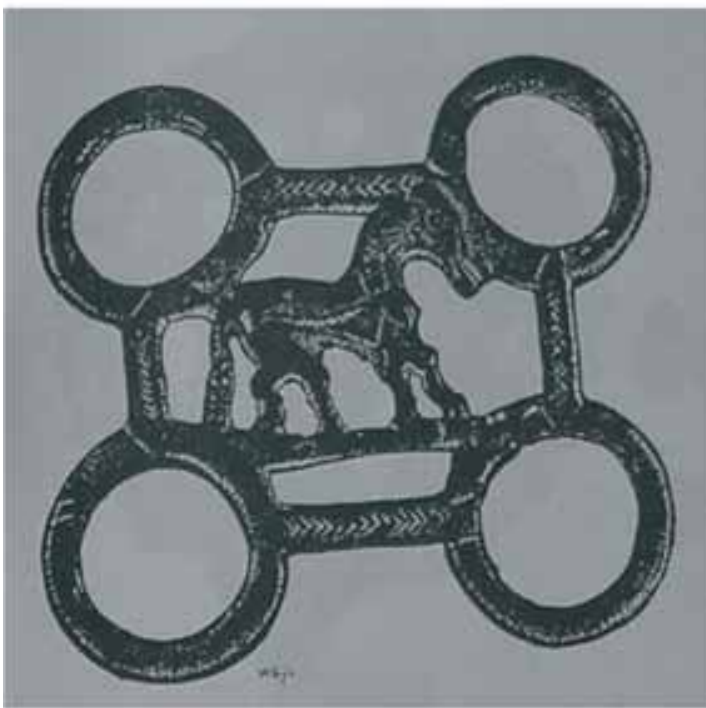

FIG. 2b-Blásquez, Historia económica, fig. 84, 1 
Est. III

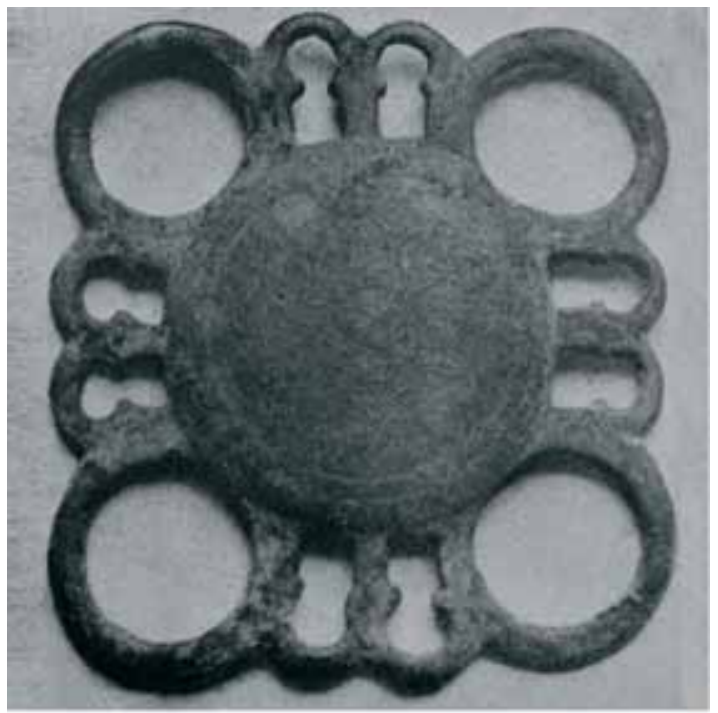

Fig. 3 - Nr. 3, da Millán, Falera romana, tav. XXII, 2

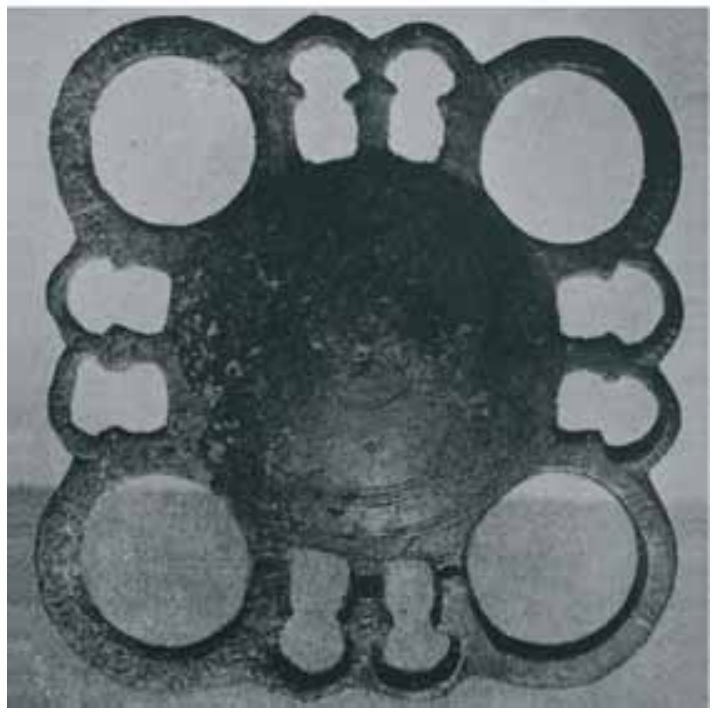

FIG. 4 -Nr. 4, da Millán, Falera romana, tav. XXII, 3 
Est. IV

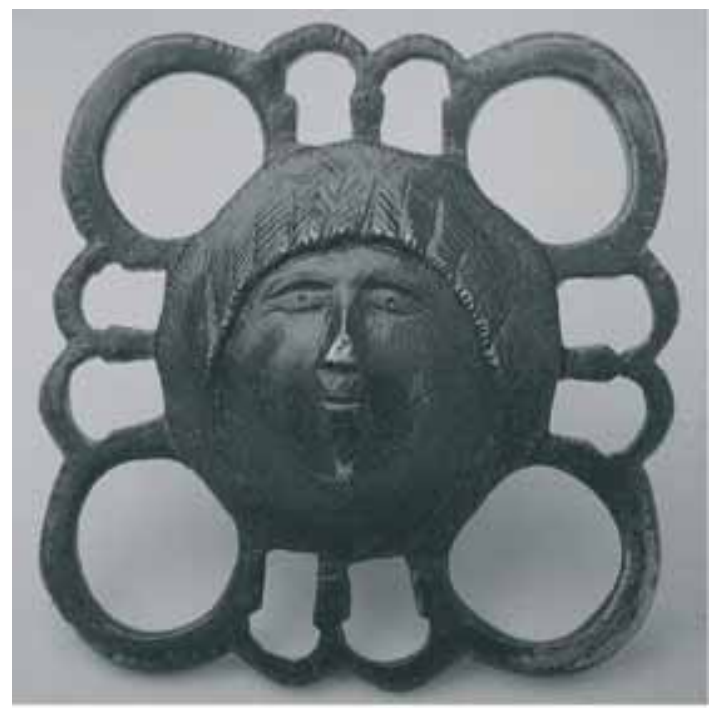

FIG. 5 - Nr. 5, da AA.VV., Bronces romanos, p. 202, $n r .73$

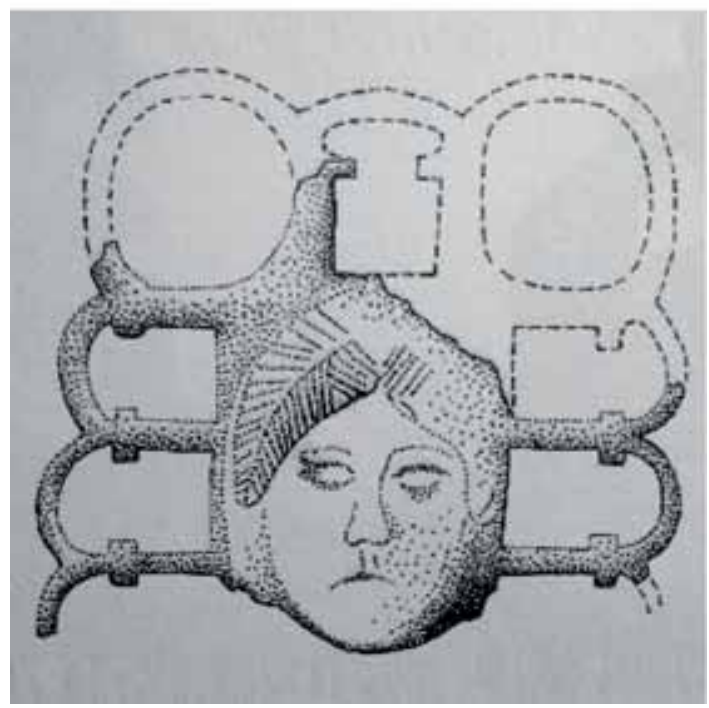

Fig. 6 - Nr. 6, da Pinto, Bronzes figurativos, nr. 293, tav. 165 
Est. V

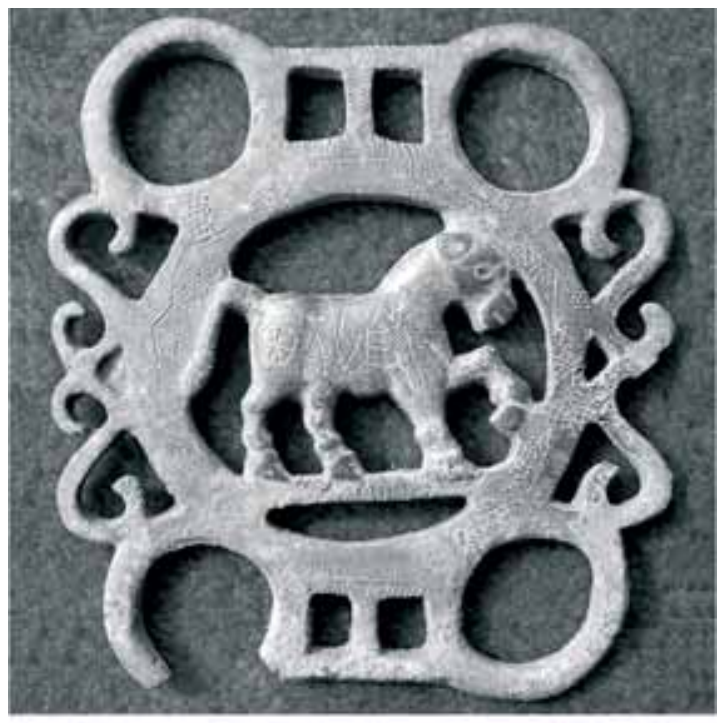

Fig. $7 \mathrm{a}-N r 7$, foto autore

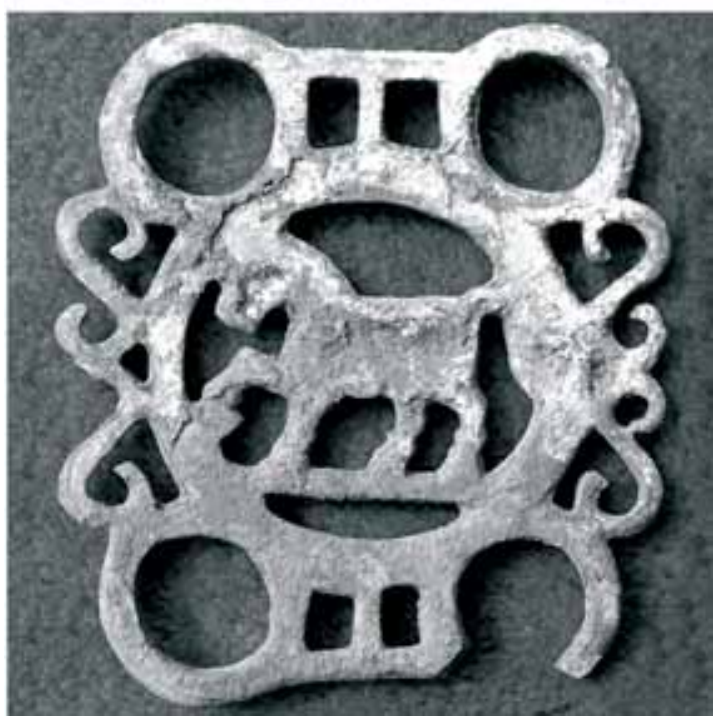

FIG. $7 \mathrm{~b}-N r$. 7, foto autore 
Est. VI

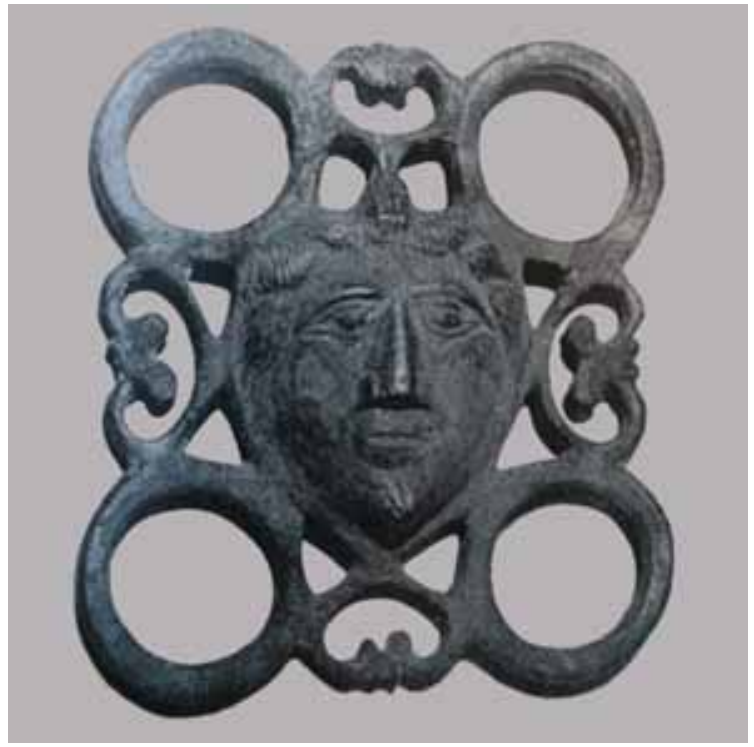

FIG. 8 - Nr. 8 Millán, Falera romana, tav. XXI, fig. 2 
EsT. VII

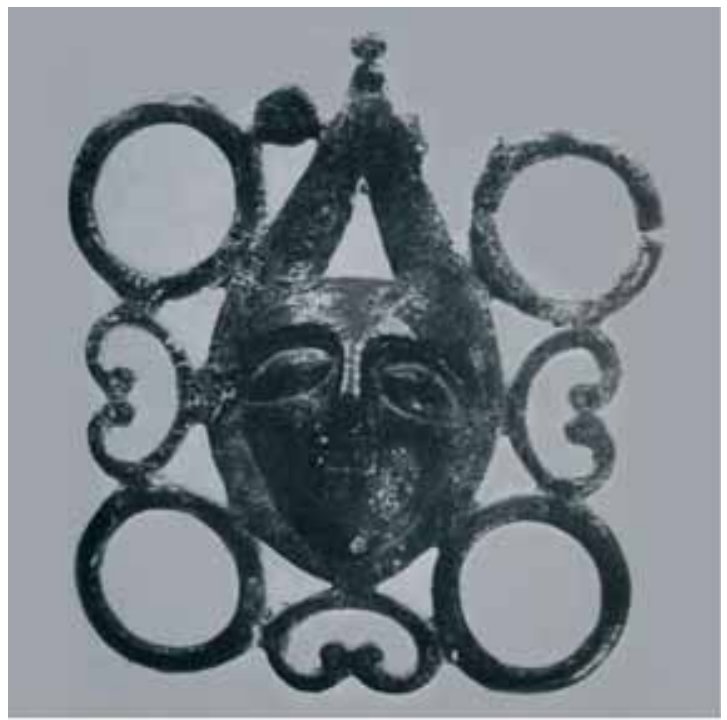

FIG. 9a - Nr. 9, da Palol, Más bronces romanos, tav. III, 2

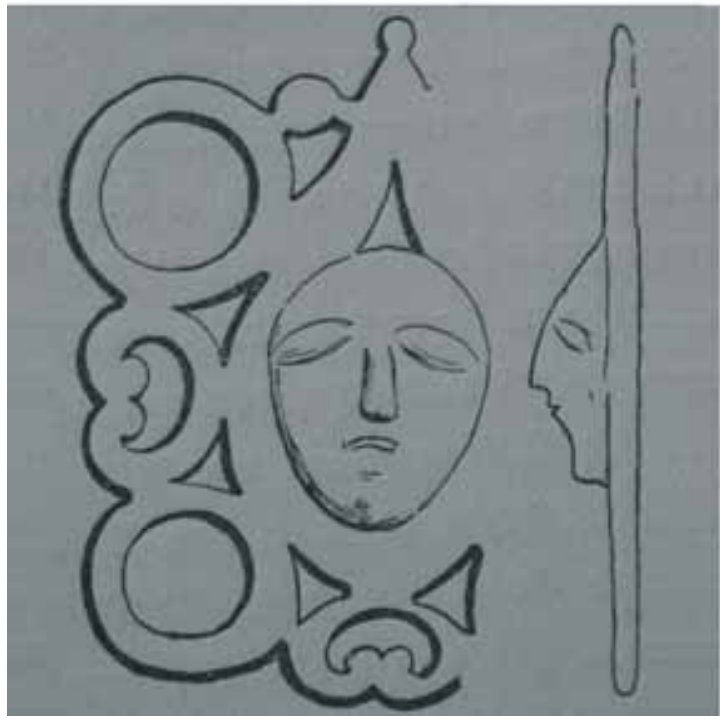

FIG. 9b-Nr. 9, da Millán, Falera romana, fig. 1 


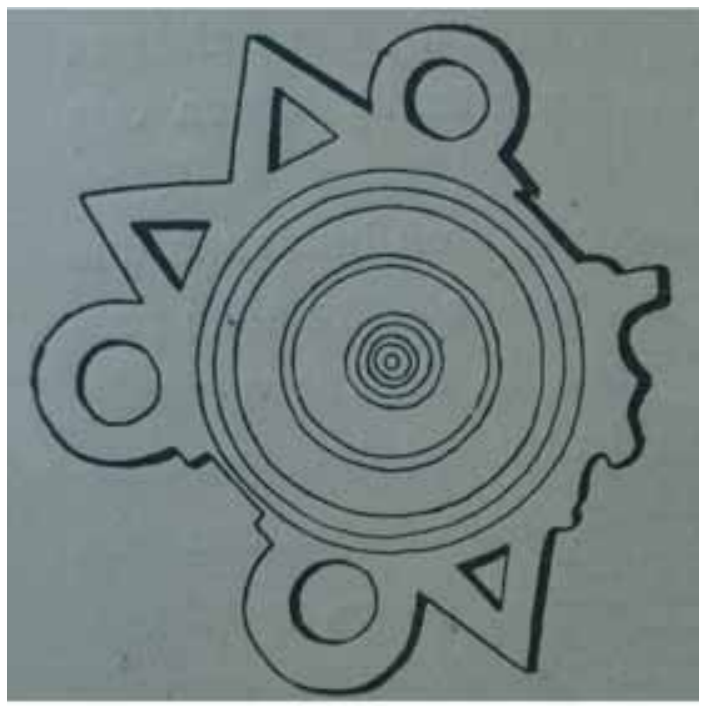

FIG. 10 - Nr. 10, da Millán, Falera romana,fig. 2

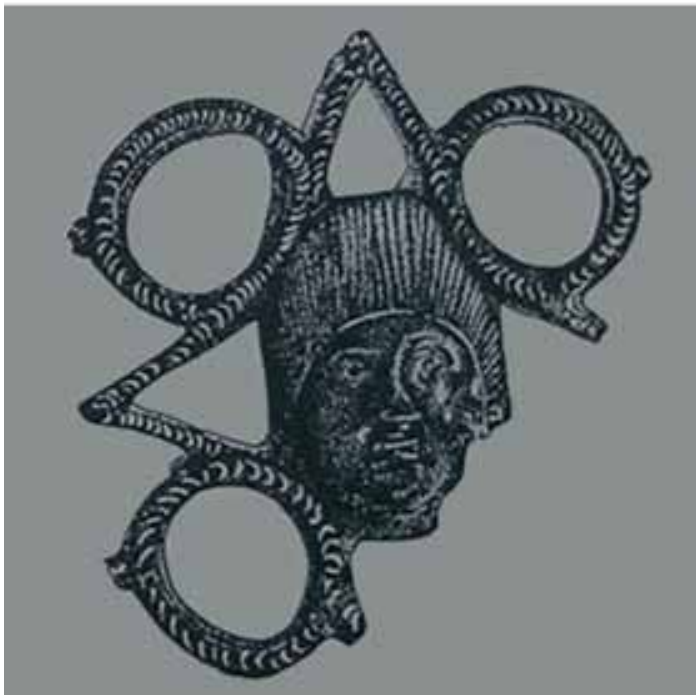

Fig. 11 - Nr. 11, da De Palol, Bronces romanos de Palencia, tav. IV, 1 
Est. IX

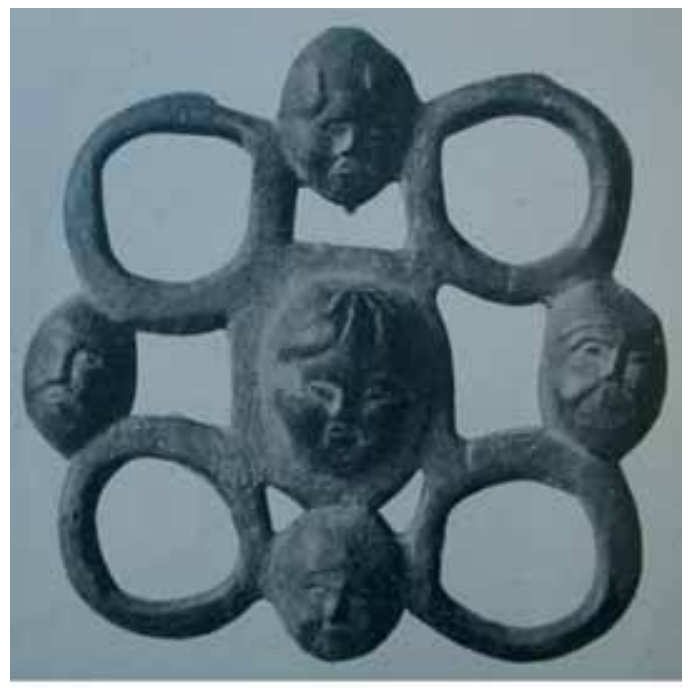

Fig. 12a - Nr. 12, da Cabré Aguiló, Tesoro, p. 289, fig. 9

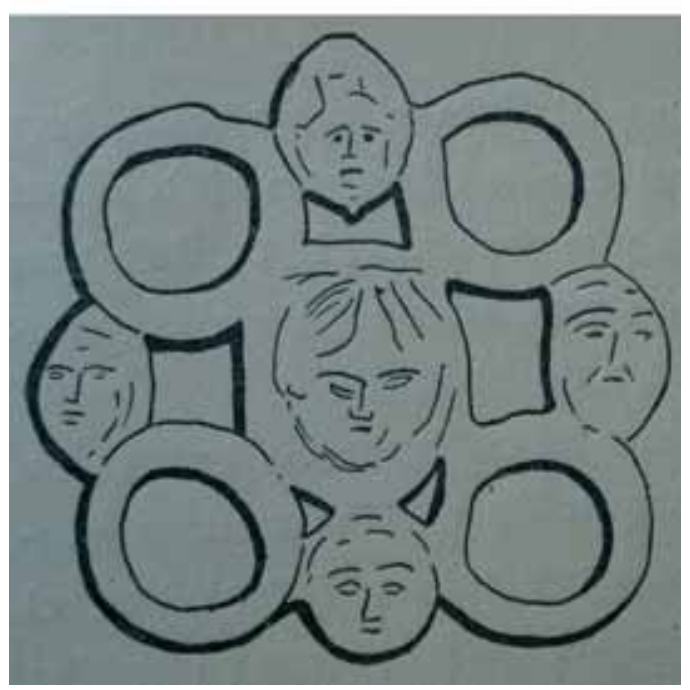

Fig. 12b-Nr. 12, da Millán, Falera romana, fig. 1 
Est. X

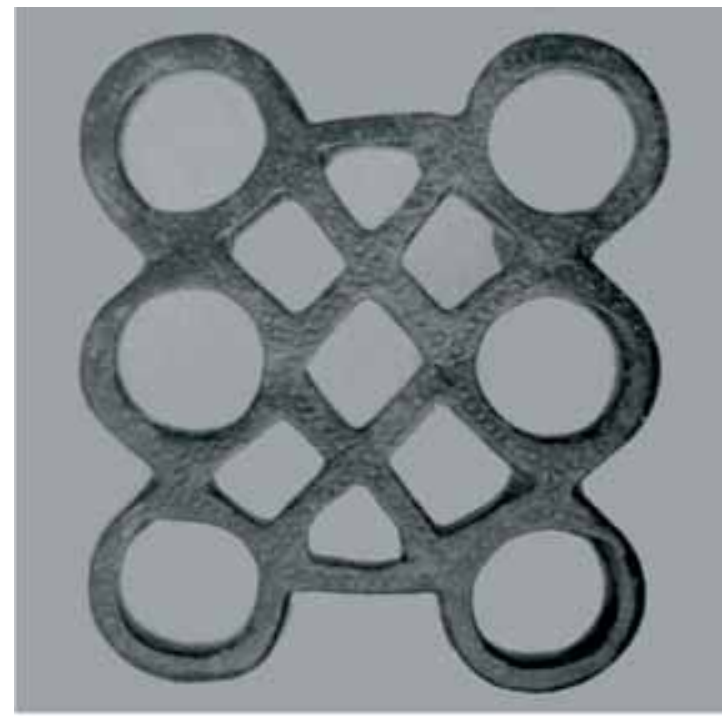

Fig. 13a-Nr. 13, da Jimeno Martínez, Argente Oliver, Gómez Santa Cruz, Villa de San Pedro, tav. VI, 2

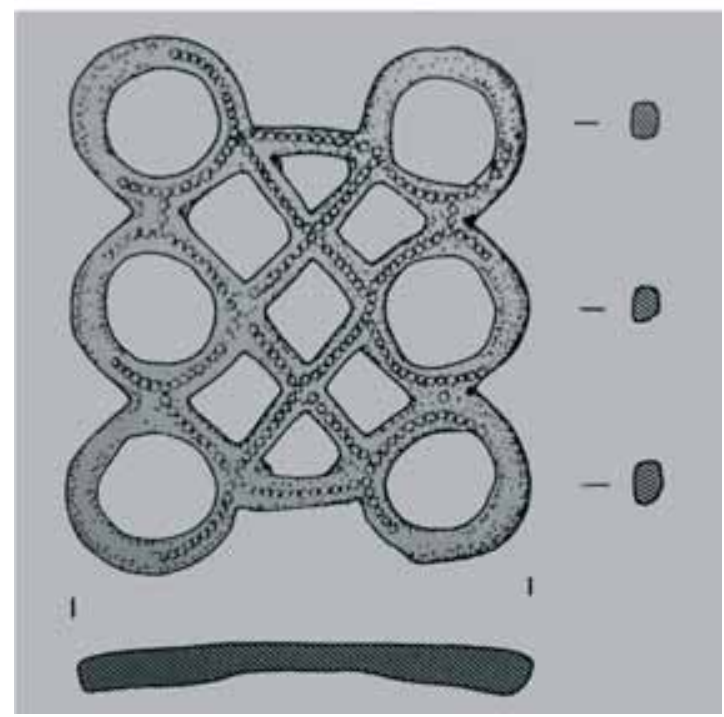

Fig. 13b - Nr. 13, da Jimeno Martínez, Argente Oliver, Gómez Santa Cruz, Villa de San Pedro, fig. 18, 241 
Est. XI

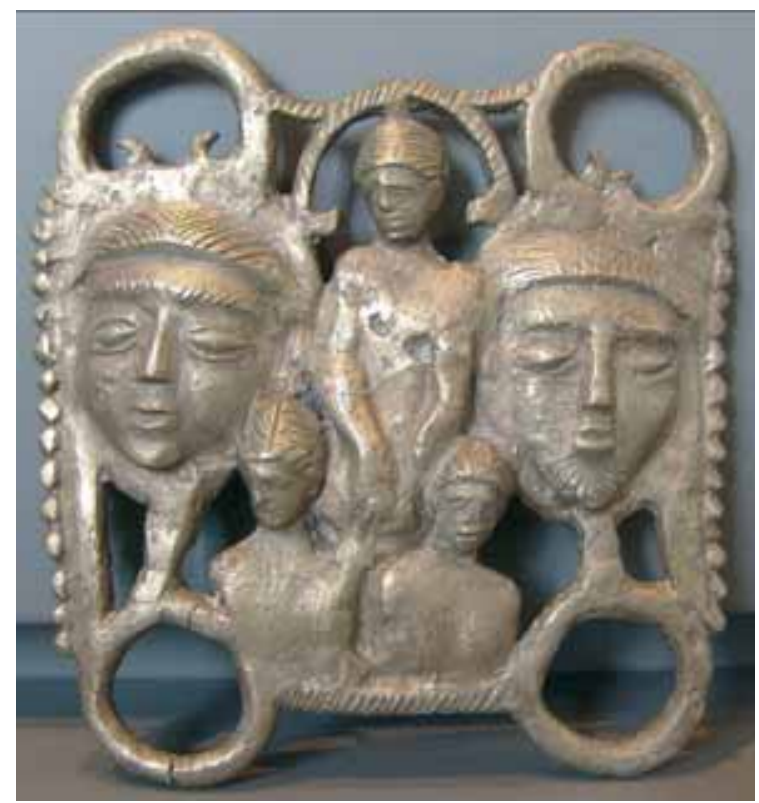

FIG. 14 - Nr. 14, per gentile concessione della Câmara Municipal de Chaves 


\section{Est. XII}

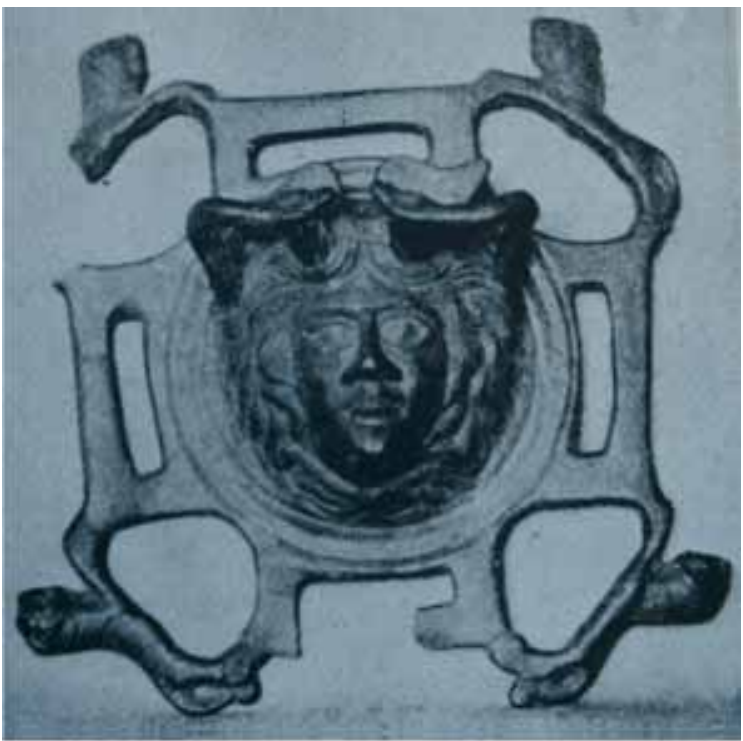

Fig. 15 - Nr. 15, da Millán, Falera romana, tav. XXI, 3

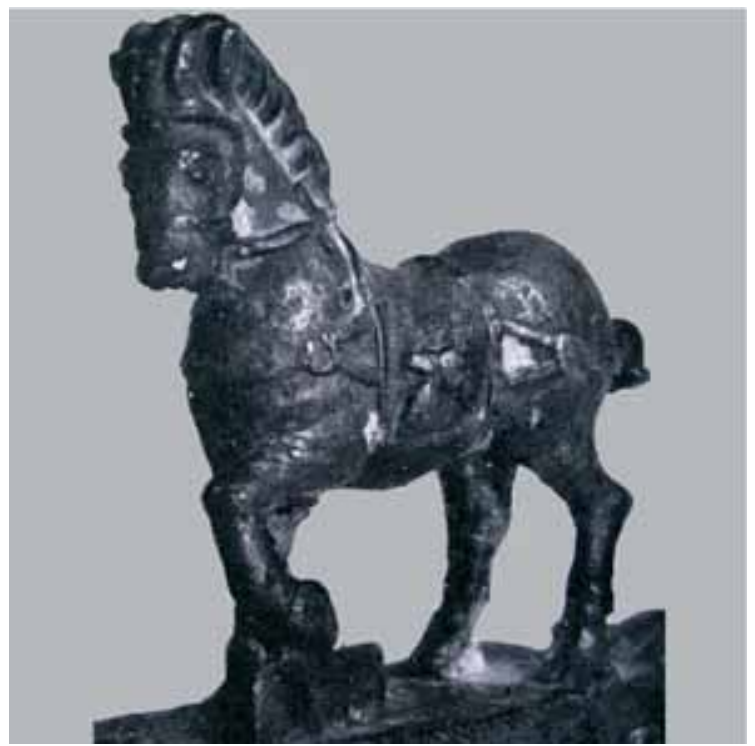

FIG. 16 - Dettaglio della sospensione di carro di Morón de la Frontera, da Blanco, Pasarriendas, fig. 5. 